\title{
Estudo Comparativo entre Toxina Botulínica e Bupivacaína para Infiltração de Pontos-Gatilho em Síndrome Miofascial Crônica *
}

\section{Comparative Study between Botulin Toxin and Bupivacaine for Trigge- ring-Points Infiltration in Chronic Myofascial Syndrome}

Eduardo Keiichi Unno ${ }^{1}$; Rioko Kimiko Sakata, TSA ${ }^{2}$; Adriana Machado Issy ${ }^{3}$

\section{RESUMO}

Unno EK, Sakata RK, Issy AM - Estudo Comparativo entre Toxina Botulínica e Bupivacaína para Infiltração de Pontos-Gatilho em Síndrome Miofascial Crônica

JUSTIFICATIVA E OBJETIVOS: Existem controvérsias sobre a eficácia da toxina botulínica em relação ao anestésico local para infiltração de pontos-gatilho. O objetivo deste estudo é comparar o efeito analgésico da toxina botulínica com o da bupivacaína, para infiltração em pontos-gatilho de síndrome miofascial crônica.

MÉTODO: Foram avaliados 20 pacientes, divididos em dois grupos. Os pacientes do $G 1(n=10)$ receberam $25 U$ de toxina botulínica e os do G2 ( $n=10)$, bupivacaína a 0,25\%, em um a três pontos-gatilho, sendo 0,5 mL por ponto. Os pacientes foram avaliados semanalmente, durante 8 semanas. Foram associados $35 \mathrm{mg}$ de orfenadrina, e $300 \mathrm{mg}$ de dipirona, a cada 8 horas, e os pacientes foram submetidos à estimulação elétrica transcutânea, duas vezes por semana, durante 1 hora por sessão. A intensidade da dor foi avaliada através da escala numérica verbal e a qualidade da analgesia, pelo paciente, nos momentos zero (antes da injeção), e após 30 minutos, 1, 2, 3, 4, $5,6,7$ e 8 semanas. Os resultados foram submetidos à análise estatística (Mann-Whitney e Exato de Fisher).

RESULTADOS: Após 30 minutos da aplicação e com 1 e 4 semanas, a intensidade da dor no $\mathrm{G} 1$ foi menor que no $\mathrm{G} 2$. Após 2, 3, 5, 7 e 8 semanas da infiltração, não houve diferença significativa entre os grupos. A qualidade da analgesia foi considerada melhor pelos pacientes do $\mathrm{G} 1$ que do G2, exceto após 2 semanas.

CONCLUSÕES: O efeito analgésico foi melhor com toxina botulínica $(25 \mathrm{U})$ que com bupivacaína a 0,25\% para infiltração de pontos-gatilho.

Unitermos: ANESTÉSICOS, Local: bupivacaína; DROGAS: toxina botulínica A; TÉCNICAS ANESTÉSICAS: infiltração de pontos-gatilho

\footnotetext{
* Recebido da (Received from) Disciplina de Anestesiologia, Dor e Terapia Intensiva da Universidade Federal de São Paulo - Escola Paulista de Medicina (UNIFESP - EPM), São Paulo, SP

1. Estagiário do Setor de Dor da Disciplina de Anestesiologia, Dor e Terapia Intensiva da UNIFESP - EPM

2. Professora Adjunta e Responsável pelo Setor de Dor da Disciplina de Anestesiologia, Dor e Terapia Intensiva da UNIFESP - EPM

3. Professora Adjunta da Disciplina de Anestesiologia, Dor e Terapia Intensiva da UNIFESP - EPM
}

Apresentado (Submitted) em 31 de maio de 2004

Aceito (Accepted) para publicação em 23 de novembro de 2004

Endereço para correspondência (Correspondence to)

Dra. Rioko Kimiko Sakata

Rua Três de Maio, 61/51 Vila Clementino

04044-020 São Paulo, SP

(C) Sociedade Brasileira de Anestesiologia, 2005

\section{SUMMARY}

Unno EK, Sakata RK, Issy AM - Comparative Study between Botulin Toxin and Bupivacaine for Triggering-Points Infiltration in Chronic Myofascial Syndrome

BACKGROUND AND OBJECTIVES: There are controversies about the efficacy of botulin toxin as compared to local anesthetics for triggering-points infiltration. This study aimed at comparing the analgesic effect of botulin toxin and bupivacaine for triggering-points infiltration in chronic myofascial syndrome. METHODS: Participated in this study 20 patients divided in two groups. $G 1$ patients $(n=10)$ received $25 U$ botulin toxin and $G 2$ patients $(n=10)$ received $0.25 \%$ bupivacaine in one to three triggering-points, being $0.5 \mathrm{~mL}$ per point. Patients were weekly evaluated for 8 weeks. Orphenadrine $(35 \mathrm{mg})$ and dipirone (300 mg) were associated every 8 hours and patients were submitted to transcutaneous electrical stimulation twice a week during 1 hour. Pain intensity was evaluated through verbal numeric scale and quality of analgesia was evaluated by patients in moments zero (before injection), 30 minutes after, and at 1, 2, 3, 4, 5, 6, 7 and 8 weeks. Results were submitted to statistical analysis (Mann-Whitney and Fisher Exact tests).

RESULTS: After 30 minutes, 1 and 4 weeks, pain intensity was lower for $\mathrm{G} 1$ as compared to G2. After 2, 3, 5, 7 and 8 weeks, there was no significant difference between groups. Quality of analgesia was considered better by $\mathrm{G} 1$ patients as compared to G2, except after 2 weeks.

CONCLUSIONS: Analgesic effect was better with botulin toxin (25U) as compared to $0.25 \%$ bupivacaine for triggering-points infiltration.

Key Words: ANESTHETICS, Local: bupivacaine; DRUGS: botulin toxin A; ANESTHETIC TECHNIQUES: triggering-points infiltration

\section{INTRODUÇÃO}

síndrome miofascial crônica é muito freqüente, ocorrenAdo em $30 \%$ a $85 \%$ dos pacientes que são atendidos em Clínicas de Dor. Pode ser aguda ou crônica, isolada ou associada a outras síndromes dolorosas e muitas vezes a dor é intensa e de difícil alívio.

Trata-se de uma condição dolorosa muscular regional, caracterizada pela ocorrência de bandas musculares tensas palpáveis, nas quais identificam-se pontos intensamente dolorosos, denominados de pontos-gatilho que, quando estimulados por palpação digital, ocasionam dor local intensa e referida à distância ${ }^{1,2}$.

Os pontos-gatilho podem ser encontrados em forma de nódulos e se desenvolvem após trauma, uso excessivo ou espasmo muscular prolongado. São decorrentes de sobrecargas dinâmicas (traumatismos, excesso de uso) ou estáticas (posturais) de atividades da vida diária e ocupacional. Aloca- 
lização desses nódulos é semelhante nos mesmos músculos de diferentes indivíduos. Esses nódulos podem ser encontrados em indivíduo sem dor e são denominados pontos-gatilho latentes.

Diversos são os tratamentos empregados para o tratamento da síndrome miofascial. Para pacientes que não obtêm analgesia com antiinflamatórios, relaxantes musculares, estimulação elétrica, acupuntura entre outras condutas ${ }^{3}$, a injeção de toxina botulínica em pontos-gatilho é uma opção.

Atoxina botulínica do tipo Aé uma neurotoxina que bloqueia a liberação de acetilcolina das terminações nervosas colinérgicas sem alterar a condução neural ou a síntese e o armazenamento de acetilcolina ${ }^{4-10}$.

O efeito da toxina botulínica ocorre em três estágios. Inicialmente a toxina botulínica liga-se aos receptores da membrana pré-sináptica da placa motora terminal, de maneira irreversível. A seguir ocorre endocitose, com liberação de parte da molécula no citoplasma das terminações nervosas e no final, ocorre desnervação muscular funcional, determinando contração muscular de forma seletiva.

A toxina botulínica não causa alteração na síntese e no armazenamento de acetilcolina ou na condução de sinais elétricos. A injeção de toxina botulínica em dose apropriada e localização correta no músculo provoca desnervação parcial e diminuição da contratura sem paralisia completa.

A toxina botulínica do tipo Aé um agente biológico obtido laboratorialmente, sendo uma substância cristalina estável, liofilizada, associada à albumina humana e utilizada, após diluição, em solução fisiológica e medida em unidades biológicas (U) definidas pela dose letal-50 (DL50) ${ }^{11}$.

A ação da toxina botulínica é observada em alguns dias a 2 semanas e a duração é de 6 semanas a 6 meses (em média de 3 a 4 meses). Durante o período de efeito mais intenso, observa-se atrofia muscular e alteração das fibras em exame histológico. Na recuperação formam-se brotos, reinervação e formação de novas placas terminais menores.

O principal risco é fraqueza muscular que pode ocorrer após injeção de dose maior que a recomendada para determinado músculo. A dose total excessiva também causa fraqueza de músculos próximos, porém com doses apropriadas injetadas em locais corretos, raramente ocorre complicação.

Não existem estudos para verificar se a toxina botulínica proporciona resultados melhores que o anestésico local.

O presente estudo visa comparar o efeito analgésico da toxina botulínica com a da bupivacaína em pontos-gatilho de pacientes com síndrome miofascial crônica.

\section{MÉTODO}

Após aprovação do estudo pelo Comitê de Ética em Pesquisa do Hospital São Paulo da Universidade Federal de São Paulo e assinatura do Termo de Consentimento, foram avaliados 20 pacientes adultos de ambos os sexos, portadores de síndrome miofascial, com dor crônica moderada ou intensa.
Os pacientes foram divididos em dois grupos de igual tamanho.

Foram excluídos do trabalho os pacientes que utilizaram opióides nas 24 horas antecedentes, que apresentavam infecção local ou coagulopatias, além dos que usavam anticoagulantes ou com hipersensibilidade a albumina e as grávidas.

Os pacientes do grupo $1(n=10)$ receberam $25 \mathrm{U}$ de toxina botulínica em solução fisiológica a $0,9 \%$. Os pacientes do grupo $2(n=10)$ receberam bupivacaína a $0,25 \%$ sem vasoconstritor.

A infiltração foi feita de forma idêntica em um a três pontos-gatilho em ambos os grupos, sendo $0,5 \mathrm{~mL}$ por ponto. Os pacientes foram avaliados semanalmente, durante 8 semanas, após a infiltração. Durante o estudo foram associados $35 \mathrm{mg}$ de orfenadrina, e $300 \mathrm{mg}$ de dipirona, a cada 8 horas. Os pacientes foram submetidos também à estimulação elétrica transcutânea, duas vezes por semana, durante 1 hora por sessão.

Os pacientes foram avaliados quanto à intensidade da dor através da escala numérica verbal onde zero significa ausência de dor e 10, a dor mais intensa, já experimentada. A qualidade da analgesia foi avaliada conforme relato do paciente, através de quatro descritores ( $E=$ excelente; $B=$ boa; $R$ $=$ regular; e $\mathrm{P}=$ péssima). A intensidade da dor e a qualidade da analgesia foram avaliadas nos momentos zero (antes da injeção), e após 30 minutos, 1, 2, 3, 4, 5, 6, 7 e 8 semanas.

Foi anotado o efeito analgésico e os possíveis efeitos colaterais da toxina botulínica. Os resultados foram submetidos à análise estatística (testes de Mann-Whitney e Exato de Fisher, $p<0,05)$.

\section{RESULTADOS}

Os grupos foram semelhantes em relação à idade, ao sexo, e ao índice de massa corpórea (IMC). A média de idade foi de 41,5 anos no $\mathrm{G} 1$ e de 51,3 anos, no G2. Nos dois grupos, dois pacientes eram do sexo masculino e oito, do feminino. A média de IMC foi de 24.8 no G1 e de 26.1, no G2.

Aintensidade da dor, antes da infiltração, foi semelhante nos dois grupos (Tabela I). Após 30 minutos da aplicação e com 1 e 4 semanas da infiltração, a intensidade da dor no G1 foi menor que no G2, sendo a diferença estatisticamente significativa (Tabela I). Após 2, 3, 5, 6, 7 e 8 semanas da infiltração, não houve diferença estatisticamente significativa entre os grupos, na intensidade da dor (Tabela I), (teste de Mann-Whitney).

A qualidade da analgesia foi considerada melhor pelos pacientes do $\mathrm{G} 1$ que do $\mathrm{G} 2$, sendo estatisticamente significativo, exceto após 2 semanas (Tabela II) (teste Exato de Fisher).

Foi observado efeito analgésico melhor no G1 até a oitava semana (M8) após a infiltração, na última avaliação. Nenhum efeito colateral foi observado nos dois grupos, durante o período de estudo. 
Tabela I - Intensidade da dor (Escala Numérica Verbal) dos Pacientes Portadores de Síndrome Miofascial (Média \pm DP

\begin{tabular}{lccc}
\hline \multirow{2}{*}{ Momentos } & \multicolumn{2}{c}{ Grupos } & $\mathrm{p}$ \\
\cline { 2 - 3 } M0 & $\mathrm{G} 1(\mathrm{n}=10)$ & $\mathrm{G} 2(\mathrm{n}=10)$ & 0,0753 \\
M30 & $5,8 \pm 1,3$ & $6,8 \pm 2,3$ & 0,0005 \\
M1 & $3,0 \pm 1,5$ & $5,8 \pm 1,2$ & 0,0039 \\
M2 & $2,3 \pm 2,2$ & $5,4 \pm 1,6$ & 0,3930 \\
M3 & $3,3 \pm 2,5$ & $4,3 \pm 1,6$ & 0,0524 \\
M4 & $3,0 \pm 2,8$ & $5,7 \pm 2,6$ & 0,0433 \\
M5 & $2,1 \pm 2,4$ & $5,0 \pm 3,1$ & 0,0630 \\
M6 & $2,2 \pm 2,3$ & $4,5 \pm 2,5$ & 0,0753 \\
M7 & $2,3 \pm 2,6$ & $4,5 \pm 2,8$ & 0,1903 \\
M8 & $2,9 \pm 2,5$ & $4,6 \pm 2,7$ & 0,0630 \\
\hline
\end{tabular}

G1: toxina botulínica; G2: bupivacaína; M0: antes da infiltração; M30: 30 minutos após injeção; M1 a M 8: 1 a 8 semanas após a infiltração; teste de Mann-Whitney, $\mathrm{p}<0,05$

Tabela II - Qualidade da Analgesia dos Pacientes $(n=20)$ Portadores de Síndrome Miofascial (\%)

\begin{tabular}{|c|c|c|c|c|}
\hline \multirow{2}{*}{ Momentos } & \multirow{2}{*}{ Grupos } & \multicolumn{2}{|c|}{ Qualidade da Analgesia } & \multirow{2}{*}{$\mathrm{p}$} \\
\hline & & $E+B$ & $R+P$ & \\
\hline \multirow{2}{*}{ M30 } & G1 & 100 & 0 & \multirow{2}{*}{$\mathrm{S}$} \\
\hline & G2 & 60 & 40 & \\
\hline \multirow{2}{*}{ M1 } & G1 & 70 & 30 & \multirow{2}{*}{$\mathrm{S}$} \\
\hline & G2 & 40 & 60 & \\
\hline \multirow{2}{*}{ M2 } & G1 & 60 & 40 & \multirow{2}{*}{ NS } \\
\hline & G2 & 70 & 30 & \\
\hline \multirow{2}{*}{ M3 } & G1 & 70 & 30 & \multirow{2}{*}{$\mathrm{S}$} \\
\hline & G2 & 30 & 70 & \\
\hline \multirow{2}{*}{ M4 } & G1 & 70 & 30 & \multirow{2}{*}{$\mathrm{S}$} \\
\hline & G2 & 30 & 70 & \\
\hline \multirow{2}{*}{ M5 } & G1 & 70 & 30 & \multirow{2}{*}{$\mathrm{S}$} \\
\hline & G2 & 40 & 60 & \\
\hline \multirow{2}{*}{ M6 } & G1 & 70 & 30 & \multirow{2}{*}{$\mathrm{S}$} \\
\hline & G2 & 40 & 60 & \\
\hline \multirow{2}{*}{ M7 } & G1 & 70 & 30 & \multirow{2}{*}{$\mathrm{S}$} \\
\hline & G2 & 40 & 60 & \\
\hline \multirow{2}{*}{ M8 } & G1 & 70 & 30 & \multirow{2}{*}{$S$} \\
\hline & G2 & 40 & 60 & \\
\hline
\end{tabular}

$E=$ excelente, $B$ : bom; $R$ : regular; $P$ : péssima; teste Exato de Fisher $p<0,05$

\section{DISCUSSÃO}

Diversos tratamentos são empregados para o alívio da dor da síndrome miofascial e, para infiltração de pontos-gatilho vários fármacos foram avaliados. Os anestésicos locais são os mais utilizados, porém como sua duração é curta, apesar do efeito ser adequado, pode ocorrer recorrência da crise de dor.

A infiltração de toxina botulínica do tipo A vem sendo realizada, com resultados analgésicos satisfatórios ${ }^{6,8,12-15}$.

Os estudos mostram que a dose segura de toxina botulínica para o tratamento da síndrome miofascial é de $4 \mathrm{U} / \mathrm{kg}^{16}$, sendo que neste estudo foram utilizadas doses menores. A dose empregada foi de $25 \mathrm{U}$, independentemente do peso, porém o paciente de menor peso tinha $41 \mathrm{~kg}$.

A toxina botulínica age diretamente no músculo afetado, provocando relaxamento e conseqüentemente, reduzindo a dor. Após a redução da intensidade da dor com o emprego da toxina botulínica, o paciente consegue realizar exercício físico, necessário para sua recuperação e melhorar sua qualidade de vida ${ }^{13}$.

É importante lembrar que no período em que o paciente estiver sob os efeitos do medicamento, deve-se intensificaro processo de reabilitação e do reequilibro muscular com medidas físicas para obter uma melhor eficácia do tratamento ${ }^{11,13}$.

Neste estudo foi observado que a toxina botulínica do tipo Aé mais eficaz que o anestésico local em infiltração de pontos-gatilho para o tratamento da síndrome miofascial. Apesar de não ter sido observada diferença estatisticamente significante nos momentos M2, M3, M5, M6, M7, M8, os escores da intensidade de dor foram menores com o uso da toxina botulínica em relação aos obtidos após anestésico local.

Embora o uso de anestésico local para infiltração de pontos-gatilho seja uma boa opção, a aplicação da toxina botulínica do tipo A pode ser uma alternativa, especialmente para pacientes portadores de síndrome miofascial de longa duração e que após infiltração de anestésico local têm alívio adequado da dor, porém com recorrência da dor. O alívio prolongado da dor é importante, pois permite relaxamento do músculo acometido durante tempo suficiente para recuperação do paciente.

A restrição do uso da toxina botulínica do tipo $A$, devido ao seu custo, em alguns pacientes pode ser compensada pelo fato de reduzir o número de aplicações (sua duração é de até seis meses). Com anestésico local pode haver necessidade de várias infiltrações, com desconforto local, além da necessidade de vários atendimentos, o que também gera custos. Nas condições deste estudo clínico o efeito analgésico foi melhor com toxina botulínica (25U) que com bupivacaína $0,25 \%$ para infiltração de pontos-gatilho. 


\section{Comparative Study between Botulin Toxin and Bupivacaine for Triggering-Points Infiltration in Chronic Myofascial Syndrome}

Eduardo Keiichi Unno, M.D.; Rioko Kimiko Sakata, TSA, M.D.; Adriana Machado Issy, M.D.

\section{INTRODUCTION}

Chronic myofascial syndrome is very common and is present in $30 \%$ to $50 \%$ of patients referred to Pain Clinics. It may be acute or chronic, isolated or associated to other painful syndromes and very often pain is severe and difficult to relief. It is a regional muscle pain characterized by palpable tense muscle bands in which severely painful points are identified, called triggering-points which, when stimulated by digital palpation, cause severe local pain and distant referred pain ${ }^{1,2}$. Triggering-points may be found as nodes and are developed after trauma, excessive use or prolonged muscle spasm. They are caused by dynamic (trauma, excessive use) or static (postural) overloads of daily and occupational activities. Location of these nodes is similar in the same muscles of different individuals. These nodes may be found in painless individuals and are called latent triggering-points.

There are several treatments for myofascial syndrome. For patients not obtaining analgesia with anti-inflammatory drugs, muscular relaxants, electric stimulation or acupuncture among other approaches ${ }^{3}$, botulin toxin injection in triggering-points may be an option.

Botulin toxin type $A$ is a neurotoxin blocking acetylcholine release from cholinergic nervous terminations without changing neural conduction or acetylcholine synthesis and storage ${ }^{4-10}$

There are three stages for botulin toxin effects. Initially, botulin toxin is irreversibly linked to pre-synaptic membrane receptors of terminal endplate. Then, there is endocytosis with release of part of the molecule in nervous terminations cytoplasm, and at the end, there is functional muscular denervation determining selective muscle contraction.

Botulin toxin does not change acetylcholine synthesis and storage or electrical signals conduction. Botulin toxin injection in adequate dose and muscle location promotes partial denervation and decreased contracture without total paralysis.

Botulin toxin type $A$ is a biological agent obtained in laboratory and is a stable, dried frozen crystalline solution associated to human albumin and used after dilution in saline. It is measured in biological units $(U)$ defined by total lethal-50 dose (DL50) ${ }^{11}$.

Botulin toxin action is observed in some days to 2 weeks and lasts for 6 weeks to 6 months (mean of 3 to 4 months). During more intense effect, muscle atrophy and fiber changes are observed at histological exam. During recovery, sprouts are formed with re-enervation and formation of new smaller endplates.

Primary risk is muscle weakness, which may be present after injection of a dose above the indication for a certain muscle. Total excessive dose may also promote weakness of neighbor muscles, however with adequate doses injected in adequate sites, complications are very uncommon.

There are no studies checking whether botulin toxin promotes better results as compared to local anesthetics.

Our study aimed at comparing the analgesic effect of botulin toxin and bupivacaine in triggering-points of chronic myofascial syndrome patients.

\section{METHODS}

After Hospital São Paulo, Universidade Federal de São Paulo Research Ethics Committee approval and their informed consent, participated in this study 20 adult patients of both genders, with myofascial syndrome and moderate or severe chronic pain. Patients were divided in two equal groups. Exclusion criteria were patients under opioids in the last 24 hours, with local infection or coagulopaties, those under anticoagulants, hypersensitive to albumin and pregnant women. Group 1 patients $(n=10)$ received $25 U$ botulin toxin in saline. Group 2 patients $(n=10)$ received $0.25 \%$ bupivacaine without vasoconstrictor. Infiltration was identically performed in one to three triggering-points in both groups, being $0.5 \mathrm{~mL}$ per point. Patients were weekly evaluated for 8 weeks. During the study, $35 \mathrm{mg}$ orphenadrine and $300 \mathrm{mg}$ dipirone were associated every 8 hours. Patients were also submitted to transcutaneous electric stimulation twice a week for 1 hour. Pain intensity was evaluated by verbal numeric scale where zero meant no pain and 10 the most severe pain ever experienced. Quality of analgesia was evaluated according to patients' reports through 4 descriptors ( $E=$ excellent; $G=$ good; $R$ = regular; $P$ = poor). Pain intensity and quality of analgesia were evaluated in moments zero (before injection), 30 minutes after injection and at 1, 2, 3, 4, 5, 6, 7, and 8 weeks.

Botulin toxin analgesic effect and possible side effects were recorded. Results were submitted to statistical analysis (Mann-Whitney and Fisher Exact tests, p < 0.05).

\section{RESULTS}

Groups were similar in age, gender and body mass index (BMI). Mean age was 41.5 years for $\mathrm{G} 1$ and 51 .3 years for $\mathrm{G} 2$. In both groups, two patients were male and 8 female. Mean BMI was 24.8 for $\mathrm{G} 1$ and 26.1 for $\mathrm{G} 2$.

Pain intensity before infiltration was similar for both groups (Table I). After 30 minutes, 1 and 4 weeks, pain intensity in G1 was lower as compared to $\mathrm{G} 2$, with statistically significant difference (Table I). After 2, 3, 5, 6, 7 and 8 weeks, there were no statistically significant differences between groups in pain intensity (Table I) (Mann-Whitney test). 
Table I - Pain Intensity (Verbal Numeric Scale) of Myofascial Syndrome Patients $(n=20)($ Mean $\pm S D)$

\begin{tabular}{lccc}
\hline \multirow{2}{*}{ Moments } & \multicolumn{2}{c}{ Groups } & $\mathrm{p}$ \\
\cline { 2 - 3 } M0 & $\mathrm{G} 1(\mathrm{n}=10)$ & $\mathrm{G} 2(\mathrm{n}=10)$ & 0.0753 \\
M30 & $5.8 \pm 1.3$ & $6.8 \pm 2.3$ & 0.0005 \\
M1 & $3.0 \pm 1.5$ & $5.8 \pm 1.2$ & 0.0039 \\
M2 & $2.3 \pm 2.2$ & $5.4 \pm 1.6$ & 0.3930 \\
M3 & $3.3 \pm 2.5$ & $4.3 \pm 1.6$ & 0.0524 \\
M4 & $3.0 \pm 2.8$ & $5.7 \pm 2.6$ & 0.0433 \\
M5 & $2.1 \pm 2.4$ & $5.0 \pm 3.1$ & 0.0630 \\
M6 & $2.2 \pm 2.3$ & $4.5 \pm 2.5$ & 0.0753 \\
M7 & $2.3 \pm 2.6$ & $4.5 \pm 2.8$ & 0.1903 \\
M8 & $2.9 \pm 2.5$ & $4.6 \pm 2.7$ & 0.0630 \\
\hline
\end{tabular}

G1: botulin toxin; G2: bupivacaine; M0: before infiltration; M30: 30 minutes after injection; M1 to M 8: 1 to 8 weeks after infiltration; Mann-Whitney test, $p<0.05$

Analgesia was considered better by $\mathrm{G} 1$ patients as compared to $\mathrm{G} 2$, with statistically significant difference, except after 2 weeks (Table II) (Fisher Exact test).

Table II - Quality of Analgesia of Myofascial Syndrome Patients $(n=20)(\%)$

\begin{tabular}{|c|c|c|c|c|}
\hline \multirow{2}{*}{ Moments } & \multirow{2}{*}{ Groups } & \multicolumn{2}{|c|}{ Quality of Analgesia } & \multirow{2}{*}{$\mathrm{p}$} \\
\hline & & $E+G$ & $R+P$ & \\
\hline \multirow{2}{*}{ M30 } & G1 & 100 & 0 & \multirow[t]{2}{*}{ S } \\
\hline & G2 & 60 & 40 & \\
\hline \multirow{2}{*}{ M1 } & G1 & 70 & 30 & \multirow[t]{2}{*}{ S } \\
\hline & G2 & 40 & 60 & \\
\hline \multirow{2}{*}{ M2 } & G1 & 60 & 40 & \multirow[t]{2}{*}{ NS } \\
\hline & G2 & 70 & 30 & \\
\hline \multirow{2}{*}{ M3 } & G1 & 70 & 30 & \multirow[t]{2}{*}{ S } \\
\hline & G2 & 30 & 70 & \\
\hline \multirow{2}{*}{ M4 } & G1 & 70 & 30 & \multirow[t]{2}{*}{$S$} \\
\hline & G2 & 30 & 70 & \\
\hline \multirow{2}{*}{ M5 } & G1 & 70 & 30 & \multirow[t]{2}{*}{ S } \\
\hline & G2 & 40 & 60 & \\
\hline \multirow{2}{*}{ M6 } & G1 & 70 & 30 & \multirow[t]{2}{*}{ S } \\
\hline & G2 & 40 & 60 & \\
\hline \multirow{2}{*}{ M7 } & G1 & 70 & 30 & \multirow[t]{2}{*}{ S } \\
\hline & G2 & 40 & 60 & \\
\hline \multirow{2}{*}{ M8 } & G1 & 70 & 30 & \multirow[t]{2}{*}{$\mathrm{S}$} \\
\hline & G2 & 40 & 60 & \\
\hline
\end{tabular}

$E$ = excellent, G: good; R: regular; P: poor; Fisher's Exact test $p<0.05$

There has been better analgesic effect in $\mathrm{G} 1$ until the $8^{\text {th }}$ week (M8) during last evaluation. No side effect was observed in both groups during the study period.

\section{DISCUSSION}

Different treatments are used to relieve myofascial syndrome pain and many drugs have been evaluated for triggering-points infiltration. Local anesthetics are the most common, however since they have short duration, notwithstanding the adequate effect there might be pain recurrence.

Botulin toxin type A infiltration is being used with satisfactory analgesic results ${ }^{6,8,12-15}$.

Studies have shown that safe botulin toxin dose to treat myofascial syndrome is $4 \mathrm{U} / \mathrm{kg}^{16}$, but our study has used lower doses. Dose was $25 \mathrm{U}$ regardless of weight, however the lightest patient weighed $41 \mathrm{~kg}$.

Botulin toxin acts directly on the affected muscle, promoting relaxation and, as a consequence, decreasing pain. When pain intensity is decreased, patients are able to perform physical exercises needed for their recovery and quality of life improvement ${ }^{13}$.

It is important to remind that during the period in which patients are under drug effects, rehabilitation and muscle re-balance processes should be intensified with physical measures to obtain more effective treatment ${ }^{11,13}$.

Our study has observed that botulin toxin type Ais more effective as compared to local anesthetics in triggering-points infiltration to treat myofascial syndrome. Although with no statistically significant difference in moments $M 2, M 3, M 5, M 6, M 7$, $M 8$, pain intensity scores were lower with botulin toxin as compared to local anesthetics.

Local anesthetics for triggering-points infiltration are a good option, but botulin toxin type A may be an alternative, especially for patients with long lasting myofascial syndrome who achieve adequate pain relief after local anesthetic infiltration but who have pain recurrence. Prolonged pain relief is important because it relaxes muscles for enough time for patients' recovery.

Although being limited by its high cost, botulin toxin A may be cost-effective for some patients because fewer applications are needed (it lasts for up to 6 months). Local anesthetics may require several infiltrations with local discomfort, in addition to the need of several medical visits, which also generates costs.

In the conditions of this clinical study, analgesic effects were better with botulin toxin ( $25 \mathrm{U}$ ) as compared to $0.25 \%$ bupivacaine for triggering-points infiltration.

\section{REFERÊNCIAS - REFERENCES}

01. Fischer AA - Pressure threshold measurement for diagnosis of myofascial pain and evaluation of treatment results. Clin J Pain, 1987;2:207-214.

02. Fricton JR - Myofascial Pain Syndrome. Characteristics and Etiology, em: Fricton JR, Award E - Advances in Pain Research and Therapy, New York, Raven Press, 1990;107-127.

03. Esenyel M, Caglar N, Aldemir T - Treatment of myofascial pain. Am J Phys Med Rehabil, 2000;79:48-52.

04. O'Brien CF - Clinical application of botulinum toxin: implication for pain management. Pain Digest, 1998;8:342-345. 
05. O’Brien CF - Injection techniques for botulinum toxin using electromyography and electrical stimulation. Muscle Nerve, 1997;6:(Suppl):S176-S180

06. Porta M, Perretti A, Gamba M et al - The rationale and results of treating muscle spasm and myofascial syndrome with botulinum toxin type A. Pain Digest, 1998;8:346-352.

07. Porta M, Perretti A, Gamba M et al - Compartment of botulinum toxin injection for myofascial pain relief. Dolor, 1997;12:42.

08. Yue SK - Initial experience in the use of botulinum toxin A for the treatment of myofascial related muscle dysfunctions. J Musculoskel Pain, 1995;3:22.

09. Porta M - A comparative trial of botulinum toxin type $A$ and methylprednisolone for the treatment of myofascial pain syndrome and pain from chronic muscle spasms. Pain, 2000;85:101-105.

10. Brashear A, Lew MF, Dykstra DD et al - Safety and efficacy of Neurobloc (botulinum toxin type B) in type A - responsive cervical dystonia. Neurology, 1999;53:1439-1446.

11. Lianza S - Consenso Nacional sobre Espasticidade. Diretrizes para Diagnósticos e Tratamentos. SBMFR, São Paulo, 2001;24-27.

12. Acquadro MA, Borodic G - Treatment of myofascial pain with botulinum A toxin. Anesthesiology, 1994;80:705-706.

13. Porta $M$ - $L$ 'impiego della tossina bolulinica tipo A nelle síndrome dolorosi miofasciali. La Riabilitazione, 1999;32:49-55.

14. Childers M, Wilson D, Galate J et al - Treatment of painful muscle syndroms with botulinum toxin: A review. J Back Musculoskel Rehabil, 1998;10:89-96.

15. Racz GB - D. A. B. P. M. Pain Digest, 1998;8:353-356.

16. Aoki R - The Development of BOTOX ${ }^{\circledR}$ - It's History and Pharmacology. Pain Digest, 1998;8:337-341.

\section{RESUMEN}

Unno EK, Sakata RK, Issy AM - Estudio Comparativo entre Toxina Botulínica y Bupivacaína para Infiltración de Puntos-Gatillo en Síndrome Miofascial Crónica

JUSTIFICATIVA Y OBJETIVOS: Existen controversias sobre la eficacia de la toxina botulínica con relación al anestésico local para infiltración de puntos-gatillo. El objetivo de este estudio es comparar el efecto analgésico de la toxina botulínica con el de la bupivacaína, para infiltración en puntos-gatillo de síndrome miofascial crónica.

MÉTODO: Fueron evaluados 20 pacientes, divididos en dos grupos. Los pacientes del G1 $(n=10)$ recibieron $25 \mathrm{U}$ de toxina botulínica y los del G2 $(n=10)$, bupivacaína a 0,25\%, de un a tres puntos-gatillo, siendo $0,5 \mathrm{~mL}$ por punto. Los pacientes fueron evaluados semanalmente, durante 8 semanas. Fueron asociados $35 \mathrm{mg}$ de orfenadrina, y $300 \mathrm{mg}$ de dipirona, a cada 8 horas, los pacientes fueron sometidos a estimulación eléctrica transcutánea, dos veces por semana, durante 1 hora por sesión. La intensidad del dolor fue evaluada a través de la escala numérica verbal y la calidad de la analgesia, por el paciente, en los momentos cero (antes de la inyección), y después de 30 minutos, 1, 2, 3, 4, 5, 6, 7 y 8 semanas. Los resultados fueron sometidos al análisis estadístico (Mann-Whitney y Exacto de Fisher).

RESULTADOS: Después de 30 minutos de la aplicación y con 1 y 4 semanas, la intensidad del dolor en el G1 fue menor que en el G2. Después de 2, 3, 5, 7 y 8 semanas de la infiltración, no hubo diferencia significativa entre los grupos. La calidad de la analgesia fue considerada mejor por los pacientes del G1 que del G2, excepto después de 2 semanas.

CONCLUSIONES: El efecto analgésico fue mejor con toxina botulínica (25U) que con bupivacaína a 0,25\% para infiltración de puntos-gatillo. 